Canadian Journal of Soil Science Revue canadienne de la science du sol

\title{
Evaluation of a logarithmic solution to the convection- dispersion equation obtained from boundary-layer theory
}

\begin{tabular}{|r|l|}
\hline Journal: & Canadian Journal of Soil Science \\
\hline Manuscript ID & CJSS-2017-0055.R1 \\
\hline Manuscript Type: & Article \\
\hline Date Submitted by the Author: & 07-Jul-2017 \\
\hline Complete List of Authors: & $\begin{array}{l}\text { Wang, Jiao } \\
\text { ji, Hengying } \\
\text { Shao, Mingan; Institute of Geographic Sciences and Natural Resources } \\
\text { Research, }\end{array}$ \\
\hline Keywords: & $\begin{array}{l}\text { solute transport, convection-dispersion equation, boundary-layer theory, } \\
\text { logarithmic function, transport-parameter estimation }\end{array}$ \\
\hline &
\end{tabular}

SCHOLARONE $^{\text {Manuscripts }}$ 
Evaluation of a logarithmic solution to the convection-dispersion equation obtained from boundary-layer theory

Jiao Wang ${ }^{2,3, \#}$, Hengying $\mathrm{Ji}^{1,, \#}$, Ming'an Shao ${ }^{1,2,3,{ }^{*}}$

${ }^{1}$ College of Natural Resources and Environment, Northwest Agricultural \& Forest University, Yangling 712100, Shannxi Province, China

${ }^{2}$ Key Laboratory of Ecosystem Network Observation and Modeling, Institute of Geographic Sciences and Natural Resources Research, Chinese Academy of Sciences, Beijing 100101, China

${ }^{3}$ University of Chinese Academy of Sciences, Beijing 100049, China

\# These authors contributed equally to this work

*Corresponding author. Email: shaoma@igsnrr.ac.cn 


\begin{abstract}
Introducing boundary-layer (BL) theory to solve problems of solute transport provides a simple and accurate alternative method to estimate transport parameters. Most BL solutions to the convection-dispersion equation (CDE) are derived from the hypothesis of a zero concentration gradient at the position of solute front, which is inconsistent with the actual situation. This study assumes a logarithmic concentration profile and presents a novel analytical solution to the equilibrium CDE. The concentration gradient at solute front for the logarithmic model is not regarded as zero. A range of parameter values was used to evaluate the accuracy of the logarithmic model based on the relative error between the logarithmic and the corresponding exact profiles, and soil-column experiments were used to examine the reliability of the model for parameter estimation. The accuracy of the new BL solution was greatly influenced by the values of the transport parameters. The logarithmic profile matched the exact profile well when the rate of change of concentration was large in shallow porous media. These findings will integrate the methodology of using BL theory to solve problems of solute transport and provide a more accurate method for some cases of solute transport.
\end{abstract}

\title{
Keywords
}

Solute transport, convection-dispersion equation, boundary-layer theory, logarithmic function, transport-parameter estimation.

\section{Introduction}

Understanding the behaviour of matter transport in porous media is of great importance, 
especially for protecting the quality of groundwater and characterising nutrient transport. Convection and hydrodynamic dispersion are the dominant mechanisms responsible for transport, and a variety of models have consequently been developed to simulate them. The convection-dispersion equation (CDE) is one of the most commonly used models. A one-dimensional CDE includes terms that describe hydrodynamic dispersion, fluid convection, and linear equilibrium adsorption:

$$
R \frac{\partial C}{\partial t}=D \frac{\partial^{2} C}{\partial x^{2}}-v \frac{\partial C}{\partial x}
$$

where $C$ is the solute concentration as a function of space and time $\left[\mathrm{g} \mathrm{cm}^{-3}\right], D$ is the dispersion coefficient $\left[\mathrm{cm}^{2} \min ^{-1}\right], v$ is the average pore-water velocity $\left[\mathrm{cm} \mathrm{min}^{-1}\right], R$ is the retardation factor, $t$ is time [min], and $x$ is distance $[\mathrm{cm}]$.

$\mathrm{CDE}$ under equilibrium conditions is still widely used to simulate the behaviour of solute transport and to estimate the transport parameters for multiple materials. Garré et al. (2010) used a homogeneous $\mathrm{CDE}$ to characterise the transport and inferred parameters for a loamy sand at a column scale. Köhne et al. (2011) developed a method based on CDE for using soil-structure information to predict the transport of chemicals. Chotpantarat et al. (2012) determined the movement of single, binary, and multi-metal systems through lateritic soil columns using the equilibrium CDE.

Determining $D$ and $R$ in the CDE model is important for describing transport. A variety of techniques have been developed to estimate the two transport parameters, including statistical and deterministic methods (Inoue et al. 2000; Jury and Sposito 1985; Liu et al. 2004a; van Genuchten and Parker 1984; Wagner and Gorelick 1986; Yamaguchi et al. 1989). Using statistical methods to estimate transport parameters will lead to non-uniqueness, and deterministic methods are only 
applicable for limited conditions. Shao et al. (1998) used a boundary-layer (BL) method to solve $\mathrm{CDE}$ and obtained an approximate analytical solution by assuming parabolic and cubic concentration profiles. The BL solution has a simple mathematical expression and can be used for estimating parameters under laboratory and field conditions. Other BL solutions have been proposed based on this solution, building on the hypothesis of different descriptive functions for the concentration profile. Liu and Shao (2004a) used a non-zero flux instead of a zero flux at the lower boundary layer and solved the equation by Laplace transformation. Liu et al. (2004b) also proposed a graphing method for determining the parameters based on two unimodal curves for concentration and time. Wei and Wang (2012) introduced an exponential expression of the concentration profile and demonstrated that the exponential model was appropriate for low-velocity solute transport.

The above BL solutions were all obtained by assuming that the derivation of concentration versus distance at the solute front was zero, which differs from actual situations. This study describes the concentration profile using a logarithmic function and presents an analytical solution to CDE. The quality of the logarithmic solution was assessed by comparison to the exact solution under various combinations of parameters, and transport parameters were introduced. The novel solution to $\mathrm{CDE}$ can be used to predict concentration profiles and estimate transport parameters. It will optimise the methodology of using BL theory to solve problems of solute transport and improve the accuracy of parameter estimation.

\section{Theory}

For the constant-flux boundary condition discussed in this paper, the appropriate initial and boundary conditions are stated as (van Genuchten and Parker 1984): 


$$
\begin{gathered}
C(x, 0)=0 \\
\left.\left(-D \frac{\partial C}{\partial x}+v C\right)\right|_{x=0^{+}}=v C_{0} \\
\left.\frac{\partial C}{\partial x}\right|_{x=\infty}=0
\end{gathered}
$$

The initial concentration of zero for all $x$ indicates that the system is free of solute before the addition of a solution with concentration $C_{0}$. The gradient $\partial C / \partial x \rightarrow 0$ at $x \rightarrow \infty$ is a boundary condition for a semi-infinite system. The same assumption provided by Shao et al. (1998) is used:

$$
C(d(t), t)=0
$$

Integrating the right term of Eq. (1) and incorporating Eq. (6) into Eq. (1) gives:

$$
\int_{0}^{d(t)}\left(D \frac{\partial^{2} C}{\partial x^{2}}-v \frac{\partial C}{\partial x}\right) d x=v C_{0}
$$

If we define $I_{\mathrm{s}}(t)$ as an integral for the solute concentration of the profile:

$$
I_{s}(t)=\int_{0}^{d(t)} C(x, t) d x
$$

then, combining Eqs. (1), (7), and (8) easily gives:

$$
I_{s}(t)=\frac{v C_{0}}{R} t
$$

We further assumed that the concentration profile could be described using a logarithmic expression:

$$
C(x, t)=a_{0}(t)+a_{1}(t) \ln \left(\frac{1}{2}+\frac{x}{d(t)}\right)
$$

A simplified expression for solute distribution can be obtained by substituting Eq. (6) into Eq. (10), which yields:

$$
C(x, t)=a_{1}(t) \ln \left(\frac{1}{3}+\frac{2 x}{3 d(t)}\right)
$$


in which $x$ should satisfy the range from 0 to $d(t)$. By using boundary-layer conditions, Eq. (4), $a_{1}(t)$ can be calculated as:

$$
a_{1}(t)=\frac{v C_{0} d(t)}{-(2 D+v d \ln 3)}
$$

An equivalent expression of Eq. (11) can accordingly be obtained:

$$
C(x, t)=\frac{v C_{0} d(t)}{2 D+v d(t) \ln 3} \ln \left(\frac{3 d(t)}{2 x+d(t)}\right)
$$

To calculate $d(t)$, incorporating Eqs. (8) and (9) gives:

$$
d(t)=\frac{v t \ln 3}{(2-\ln 3) R}+\sqrt{\left(\frac{v t \ln 3}{(2-\ln 3) R}\right)^{2}+\frac{4 D t}{(2-\ln 3) R}}
$$

The logarithmic solution of a one-dimensional equilibrium CDE thus contains Eqs. (13) and (14). Three traditional transport parameters $(v, D$, and $R)$ are included in both the $\mathrm{BL}$ and analytical solutions. The average pore-water velocity, $v$, is constant when solute transport is under steady-flow conditions and can be determined by a miscible-displacement technique. $D$ depends on $v$ and is defined as:

$$
D=\lambda v
$$

where $\lambda$ is the dispersivity $[\mathrm{cm}] . \lambda$ has traditionally been viewed as a characteristic of the entire medium (Bear 1972; Fried and Combarnous 1971). The values of $\lambda$ range from 0.1 to $2 \mathrm{~cm}$ for homogeneous soil columns (Fried and Fried 1975), and those for undisturbed column are one or several orders of magnitude higher (Huang et al. 1995; Perfect et al. 2002). $R$ is referred to as the retardation factor accounting for linear equilibrium sorption or exchange. When $R=1$, the chemical and the soil do not interact (van Genuchten et al. 1986).

\section{Methods}

Evaluation of the models 
For describing volume-averaged or resident concentrations in semi-infinite soil profiles, the analytical solution of Eq. (1) is (Lindstrom et al. 1967):

$$
\begin{aligned}
\frac{C(x, t)}{C_{0}} & =\frac{1}{2} \operatorname{erfc}\left[\frac{R x-v t}{2(D R t)^{0.5}}\right]+\left(\frac{v^{2} t}{\pi D R}\right)^{\frac{1}{2}} \exp \left[\frac{-(R x-v t)^{2}}{4 D R t}\right] \\
& -\frac{1}{2}\left(1+\frac{v x}{D}+\frac{v^{2} t}{D R}\right) \exp \left(\frac{v x}{D}\right) \operatorname{erfc}\left[\frac{R x+v t}{2(D R t)^{0.5}}\right]
\end{aligned}
$$

In addition, assuming a cubic polynomial concentration profile yields a solution to the same solute-transport problem (Shao et al. 1998):

$$
\begin{gathered}
C(x, t)=\frac{v C_{0} d(t)}{3 D+v d(t)}\left(1-\frac{x}{d(t)}\right)^{3} \\
d(t)=\frac{2 v t}{R}+\sqrt{\left(\frac{2 v t}{R}\right)^{2}+\frac{12 D t}{R}}
\end{gathered}
$$

Eq. (16) has good accuracy and range of application for predicting the concentration profile.

The quality of the logarithmic model was evaluated based on a comparison to the exact solution obtained by setting multiple values for the three parameters. One hundred points with uniformly spaced intervals for each case were selected for discrepancy analysis. Relative root mean square error (RRMSE) was used to quantify the discrepancy between the logarithmic BL solutions and the analytical solution:

$$
R R M S E=\frac{\sqrt{\sum_{1}^{n}\left(y_{i}-\hat{y}\right)^{2} / n}}{\bar{y}}
$$

Where $y_{i}$ is the relative concentration calculated from the logarithmic solution, $\hat{y}$ is the corresponding exact relative concentration, and $\bar{y}$ is the mean relative concentration obtained from the exact solution.

Estimation of transport parameters 
With $d^{2}(t) / t$ as the independent variable, an equivalent expression of Eq. (14) is then:

$$
\frac{d^{2}(t)}{t}=\frac{2.44 v}{R} d(t)+\frac{4.44 D}{R}
$$

Similarly, Eq. (17) can be written as:

$$
\frac{d^{2}(t)}{t}=\frac{4 v}{R} d(t)+\frac{12 D}{R}
$$

$D$ and $R$ can be calculated by linear-fitting Eq. (19) or (20) to the data for the advance of the solute front over time. The position of the solute front can easily be measured. Flury and Flühler (1995) characterised Brilliant Blue FCF as a tracer in a study of solute transport and verified its applicability in laboratory and field experiments. Moreover, Ward et al. (1994) verified the feasibility of using time domain reflectometry (TDR) to analyse solute transport.

\section{Data sources and fitting}

Data from soil-column experiments conducted by Zheng (2001) and Wen (2005) were used to evaluate the logarithmic model. For these studies, aeolian sandy soil was collected from the Loess Plateau in northwestern China, and paddy soil was collected from Hunan province in south-central China. The experimental setup and properties of the soils are given in Table 1. A NaCl solution was used to displace deionised water in soil pores. and horizontally installed TDR probes were used to determine the solute front based on the change of electrical conductivity. $D$ and $R$ for each soil column could be estimated by linear-fitting methods. $D$ and $R$ were also estimated by fitting the breakthrough curves with the nonlinear least-squares parameter-optimisation method CXTFIT (Toride et al. 1995). The transport parameters obtained from the two approaches were compared to evaluate the quality of the logarithmic model.

\section{Results and discussion}

Response of model accuracy to transport parameters 
The prediction accuracy of the logarithmic model was variable for concentration profiles under different conditions of solute transport (Table 2). Three cases had minimum relative errors: $v=2 \mathrm{~cm} / \mathrm{h}, R=1$, and $\lambda=1 \mathrm{~cm} ; v=2 \mathrm{~cm} / \mathrm{h}, R=50$, and $\lambda=1 \mathrm{~cm}$; and $v=20 \mathrm{~cm} / \mathrm{h}, R=50$, and $\lambda$ $=10 \mathrm{~cm}$. The logarithmic model had the largest discrepancy when $v=20 \mathrm{~cm} / \mathrm{h}, R=1$, and $\lambda=1$ $\mathrm{cm}$. The transport parameters had a complex influence on model accuracy.

The average pore-water velocity $(v)$ was positively correlated with the relative error when both $R$ and $\lambda$ were set at 1 . The prediction accuracy for the concentration profile, however, first increased and then decreased for other cases when $R=1$ and for cases when $\lambda=1$ and $R=20$ or 50. The accuracy of the model increased as $\lambda, R$, and $v$ increased. These results indicated that the logarithmic model predicted the concentration profiles better for lower $v$ when at least one of $R$ and $\lambda$ were set at a small value and for higher $v$ when both $R$ and $\lambda$ were large.

Fig. 1 shows the relative concentrations at a time of $3 \mathrm{~h}$ derived from the exact solution of $\mathrm{CDE}$, the logarithmic model, and the polynomial model with varying $v$, using a case of $\lambda=10$ and $R=1$ as an example. The solute front advanced farther in the profile as $v$ increased. The concentration profiles obtained by the BL methods had the smallest and largest discrepancies when $v=0.2$ and $20 \mathrm{~cm} / \mathrm{h}$, respectively. The logarithmic profiles matched the corresponding exact profiles well when $v$ was low, indicating the reliability of the new model.

The accuracy of the logarithmic model also varied with $\lambda$. The relative errors in Table 2 indicate that the accuracy of the logarithmic model increased with $\lambda$ when $v$ was set at 0.02 or 0.2 $\mathrm{cm} / \mathrm{h}$. The prediction accuracy of the logarithmic model, however, first increased and then decreased when $v=0.2 \mathrm{~cm} / \mathrm{h}$ and $R=1$. The logarithmic model predicted the concentration profile better at higher $\lambda$ when solute transport had an average velocity of $20 \mathrm{~cm} / \mathrm{h}$. Variation was similar 
when $v=2 \mathrm{~cm} / \mathrm{h}$ and $R=1$, but the model accuracy was negatively correlated with $\lambda$ at a given $v$ and $R$ for the other cases in Table 2 for $v=2 \mathrm{~cm} / \mathrm{h}$.

Fig. 2 depicts the concentration profiles obtained using the three models with multiple $\lambda$ at $v$ $=2 \mathrm{~cm} / \mathrm{h}$ and $R=1$. The difference between the logarithmic and exact profiles decreased greatly when $\lambda$ increased from 1 to $10 \mathrm{~cm}$ and then remained small with increasing $\lambda$. Combined with the relative errors (Table 2) for all four cases, this result also indicated that the accuracy of the logarithmic model increased with $\lambda$ but tended to stabilise when $\lambda$ was $>10 \mathrm{~cm}$.

Three values of $R$ were used to analyse its influence on model accuracy (Table 2). Model accuracy improved at any given $\lambda$ when $R$ increased for $v=20 \mathrm{~cm} / \mathrm{h}$ and varied inversely for $v=$ $0.02 \mathrm{~cm} / \mathrm{h}$. The relative errors of the logarithmic model increased with $R$ when $v=0.2 \mathrm{~cm} / \mathrm{h}$, except for $\lambda=1 \mathrm{~cm}$, the error of which first decreased and then increased. Model accuracy for $v=$ $2 \mathrm{~cm} / \mathrm{h}$ increased with $R$ for $\lambda \leq 10 \mathrm{~cm}$ and decreased with increasing $R$ for $\lambda>10 \mathrm{~cm}$.

The effect of $R$ on the prediction accuracy of the BL model is also illustrated in Fig. 3. The values of $v$ and $\lambda$ were set at $2 \mathrm{~cm} / \mathrm{h}$ and $1 \mathrm{~cm}$, respectively. Each logarithmic profile and its corresponding exact profile were similar, implying that the logarithmic model could accurately predict the solute-concentration profile during transport for both conserved and retarded solutes. The relative errors paralleling each case in Table 2 also indicated a small difference between the two profiles.

The above analysis indicated that the accuracy of the logarithmic model was complexly affected by all three parameters, i.e. $v, D$, and $R$. The logarithmic model describes a concentration profile with large decreasing rate for small depth. When the three parameters determined a fast speed of solute displacement, the solute concentration in the fore part of profile will reach $C_{0}$ in a 
short period and the change rate will be low which leads to difference with the exact solution. Therefore, the logarithmic model generally matched the exact solution better when the rate of change of the solute concentration in the profile constantly decreased. The position of the solute front, however, was lower for the logarithmic than the exact solution for the given parameters.

Variation in the accuracy of the logarithmic model over time

Two average pore-water velocities were used to analyse the change of the prediction error of the logarithmic model over time (Fig. 4). Transport times ranging from 1 to $50 \mathrm{~h}$ were analysed for the case of $v=0.2 \mathrm{~cm} / \mathrm{h}$. The difference between the logarithmic and exact profiles decreased with transport until $5 \mathrm{~h}$, but the prediction error continued to increase after $5 \mathrm{~h}$, even though the relative errors before $10 \mathrm{~h}$ differed little. The prediction accuracy for the concentration profiles increased throughout the entire process when $v$ was set at $2 \mathrm{~cm} / \mathrm{h}$. The logarithmic model therefore generally predicted concentration profiles less accurately over time. Furthermore, the relative error was larger at $v=2$ than at $0.2 \mathrm{~cm} / \mathrm{h}$ at both 1 and $10 \mathrm{~h}$, implying a higher accuracy at a lower $v$. The concentration profile may deviated from the logarithmic function hypothesis over time, leading to lower accuracy of the logarithmic model for latter stage of the transport process.

Calibration of depth of solute front for the logarithmic model

Estimating transport parameters is one of the main uses of the logarithmic model. Using the model to obtain $D$ and $R$ depends on Eq. (18), so that the accurate determination of $d(t)$ is critical. The comparison in a previous section indicated that the position of the solute front was lower when calculated by the logarithmic model than by the exact distance. Calibrating the measured position of the solute front is therefore necessary before estimating the transport parameters.

Measured pore-water velocities and an $R$ of 1 for a conserved solute were used to determine 
the depth of the solute front using both the logarithmic and exact solutions. The time was in accordance with the column experiment, and $D$ was assigned values from 0.5 to $10 \mathrm{~cm}^{2} / \mathrm{h}$. The sensitivities of TDR used in the soil-column experiment, however, were 6 and $2 \%$ for detecting the solute front in the aeolian sandy soil and the paddy soil, so 0.06 and 0.02 , respectively, were regarded as the relative concentrations at the solute front for the exact solution.

The values of $d(t)$ calculated by the logarithmic model and the exact solution using the above parameters are shown in Fig. 5. The coefficients of determination $\left(R^{2}\right)$ for the aeolian sandy soil and the paddy soil were 0.9653 and 0.9095 , respectively, indicating a good linear relationship between the depths obtained by the logarithmic and exact solutions. The linear-regression equations could therefore be used for calibrating measured positions of the solute front.

Estimation of transport parameters

The calibrated data for the position of the solute front were linear-fitted for the two soils using Eq. (18) (Fig. 6). The discrepancy between the logarithmic concentration profile and the exact profile increased with the progress of the solute transport, so only the first three pairs of measured data were used to estimate the parameters. $R^{2}$ was $>0.9$ for both soils, indicating good linear relationships between $d^{2}(t) / t$ and $d(t)$. The linear-regression equations for the fitting were thus able to estimate $D$ and $R$. Table 3 lists the values of $D$ and $R$ calculated by the logarithmic and exact solutions. The two parameters for the aeolian sandy soil were both lower when estimated by the logarithmic model than by CXTFIT. The relative errors for $D$ and $R$ were 49.83 and $6.12 \%$, respectively. $D$ and $R$ for the paddy soil were 0.68 - and 0.14 -fold higher, respectively, for the logarithmic model than the exact solution. These results indicate that the logarithmic model may be better for estimating $R$ than $D$. The estimation errors for transport parameters were mainly 
caused by instrument and observation during measurement and because the BL solution is an approximate solution. These two parameters have also been estimated using a polynomial solution (Wen 2005; Zheng 2001), and the values are also listed in Table 3. The relative error of the polynomial solution for estimating $D$ for the aeolian soil was $41.85 \%$, less than that calculated by the logarithmic solution. Using a polynomial solution to estimate $R$ produced a 1.62 -fold error, indicating that the logarithmic solute was better for estimating $R$. For the paddy soil, both $D$ and $R$ were more accurately estimated by logarithmic model. The logarithmic solution was therefore able to accurately estimate $D$ and $R$, but a cubic polynomial solution may be better for estimating $D$ in some cases.

The concentration profiles obtained by the BL and exact solutions for the two soils at different times are shown in Fig. 7. The transport parameters used to plot the BL and exact profiles were from the BL method and CXTFIT, respectively. The velocity of solute transport was much higher for the paddy than the aeolian soil, so that the range of time was smaller for the paddy soil in this part of the analysis. The logarithmic model predicted the concentration profiles better for the aeolian than the paddy soil throughout the transport. This result further confirmed that the BL solution was better for slow transport speeds. The discrepancy between the BL profiles and the corresponding exact profile increased over time for each soil. The two BL models also performed differently for predicting the concentration profiles. The logarithmic model was more accurate than the polynomial model for describing concentration distribution in the soil profile and for estimating the solute-transport parameters.

\section{Conclusions}

We proposed a logarithmic solution to $\mathrm{CDE}$ without assuming a zero rate of concentration 
variation at the solute front. The effects of $v, D, R$, and $t$ on the accuracy of the logarithmic model for predicting concentration profiles were analysed based on RRMSE by giving them multiple values. Data for the depth of advancement of the solute front over time from two soil-column experiments were used to evaluate this novel solution for estimating transport parameters.

The three transport parameters $(v, D$ and $R)$ had complex influences on the accuracy of the logarithmic model. The value of $v$ had positive effect on model accuracy only when both $\lambda$ and $R$ were large and negatively influenced the model accuracy for other cases. The relative error generally increased with $\lambda$ for a lower value of $v$ but varied inversely with $\lambda$ for larger $v$. The effect of $R$ was more complicated than for $v$ and $\lambda$. A larger $R$ was more likely to increase the deviation from the exact solution for predicting concentration profiles. The match between the logarithmic profile the corresponding exact profile varied over time. The discrepancy between the two solutions increased over time. Using the data collected from the early part of the process therefore tended to increase the accuracy of the estimates of the transport parameters.

$D$ and $R$ could be determined by linear-fitting based on the calibrated data for the depth of the solute front over time. The logarithmic model was better than the polynomial model for estimating $R$. The concentration profiles obtained by the logarithmic model were also more similar to those obtained by the corresponding exact profile, indicating an advantage of our novel solution for predicting concentration profiles and estimating transport parameters.

The logarithmic solution to CDE supplements the methodology of using the BL method to solve problems of solute transport. The new solution provides a more accurate alternative for solute transport at higher pore-water velocities.

\section{Acknowledgement}


This study was financially supported by the National Natural Science Foundation of China (No. 41571130081 and 41530854).

\section{References}

Bear, J. 1972. Dynamic of Fluid In Porous Media. Soil Science 120(2):174-175.

Chotpantarat, S., Ong, S. K., Sutthirat, C. and Osathaphan, K. 2012. Competitive modeling of sorption and transport of $\mathrm{Pb}^{2+}, \mathrm{Ni}^{2+}, \mathrm{Mn}^{2+}$ and $\mathrm{Zn}^{2+}$ under binary and multi-metal systems in lateritic soil columns. Geoderma 189-190:278-287.

Flury, M. and Flühler, H. 1995. Tracer Characteristics of Brilliant Blue FCF. Soil Science Society of America Journal 59(1):22-27.

Fried, J. J. and Combarnous, M. A. 1971. Dispersion in Porous Media. Advances in Hydroscience 7:169-282.

Fried, J. J. and Fried, J. J. 1975. Groundwater pollution : Theory, methodology, modelling and practical rules. Elsevier Scientific Publishing Co, Amsterdam.

Garré, S., Koestel, J., Günther, T., Javaux, M., Vanderborght, J. and Vereecken, H. 2010. Comparison of Heterogeneous Transport Processes Observed with Electrical Resistivity Tomography in Two Soils. Vadose Zone Journal 9(2):336.

Huang, K., Toride, N. and Van Genuchten, M. T. 1995. Experimental investigation of solute transport in large, homogeneous and heterogeneous, saturated soil columns. Transport in Porous Media 18(3):283-302.

Inoue, M., Šimůnek, J., Shiozawa, S. and Hopmans, J. W. 2000. Simultaneous estimation of soil hydraulic and solute transport parameters from transient infiltration experiments. Advances in Water Resources 23(7):677-688. 
Jury, W. A. and Sposito, G. 1985. Field Calibration and Validation of Solute Transport Models for the Unsaturated Zone. Soil Science Society of America Journal 49:1331-1341.

Köhne, J. M., Schlüter, S. and Vogel, H.-J. 2011. Predicting Solute Transport in Structured Soil Using Pore Network Models. Vadose Zone Journal 10(3):1082.

Lindstrom, F. T., Haque, R., Freed, V. H. and Boersma, L. 1967. The movement of some herbicides in soils. Linear diffusion and convection of chemicals in soils. Environmental Science \& Technology 1(7):561-5.

Liu, C. P., Shao, M. A., Lv, D. Q., Xia, W. S. and Cai, S. B. 2004a. An Analytical Solution for Advance of Solute Front in Soils. Pedosphere 14(3):339-346.

Liu, C. P., Ye, L. A. and Shao, M. A. 2004b. Graphing method for parameter estimation of CDE of solute transport in soils. Acta Pedologica Sinica 41(5):720-726.

Perfect, E., Sukop, M. C. and Haszler, G. R. 2002. Prediction of Dispersivity for Undisturbed Soil Columns from Water Retention Parameters. Soil Science Society of America Journal 66:696-701.

Shao, M., Horton, R. and Miller, R. K. 1998. An approximate solution to the convection-dispersion equation of solute transport in soil. Soil Science 163(5):339-345.

Toride, N., Leij, F. and Van Genuchten, M. T. 1995. The CXTFIT code for estimating transport parameters from laboratory or filed tracer experiments. US Salinity Laboratory Riverside.

van Genuchten, M. T. and Parker, J. C. 1984. Boundary Conditions for Displacement Experiments through Short Laboratory Soil Columns. Soil Science Society of America Journal 48:703-708. van Genuchten, M. T., Wierenga, P. J. and Klute, A. 1986. Solute dispersion coefficients and retardation factors. Methods of Soil Analysispartphysical \& Mineralogical Methods. 
Wagner, B. J. and Gorelick, S. M. 1986. A Statistical Methodology for Estimating Transport Parameters: Theory and Applications to One-Dimensional Advectivec-Dispersive Systems. Water Resources Research 22(8):1303-1315.

Ward, A. L., Elrick, D. E. and Kachanoski, R. G. 1994. Laboratory Measurements of Solute Transport Using Time Domain Reflectometry. Soil Science Society of America Journal 58:1031-1039.

Wei, F. and Wang, Q. 2012. Exponential function model of soil solute transport. Res Soil Water Conserv 19(5):230-234.

Wen, H. 2005. An Experimental Study on Solute Front Transport in Soil and Parameter Estimate. Hunan Normal University.

Yamaguchi, T., Yokosi, S. and Moldrup, P. 1989. Using Breakthrough Curves for Parameter Estimation in the Convection-Dispersion Model of Solute Transport. Soil Science Society of America Journal 53:1635-1641.

Zheng, J. Y. 2001. Boundary layer method for solute transport in soils Master. Northwest A\&F University.

\section{Figure Captions}

Fig. 1 Relative concentration profiles derived from the different models at $\lambda=10 \mathrm{~cm}$ and $R=1$.

The values of $v$ are: (a) $0.02 \mathrm{~cm} / \mathrm{h}$, (b) $0.2 \mathrm{~cm} / \mathrm{h}$, (c) $2 \mathrm{~cm} / \mathrm{h}$, and (d) $20 \mathrm{~cm} / \mathrm{h}$.

Fig. 2 Relative concentration profiles obtained by the different models at $v=2 \mathrm{~cm} / \mathrm{h}$ and $R=1$. The values of $\lambda$ are: (a) $1 \mathrm{~cm}$, (b) $10 \mathrm{~cm}$, (c) $20 \mathrm{~cm}$, and (d) $50 \mathrm{~cm}$.

Fig. 3 Relative concentration profiles obtained using the different models at $v=2 \mathrm{~cm} / \mathrm{h}$ and $\lambda=10$ $\mathrm{cm}$. The values of $R$ are: (a) 1, (b) 10, (c) 20, and (d) 50 .

Fig. 4 The variation of relative root mean square error with time. (a) Aeolian sandy soil, and (b) 
paddy soil.

Fig. 5 Linear relationship between values of depth obtained from the logarithmic model and the exact solution. (a) Aeolian sandy soil, and (b) paddy soil.

Fig. 6 Linear fitting the data for the advance of the solute front over time using Eq. (18). (a) Aeolian sandy soil, and (b) paddy soil.

Fig. 7 Concentration profiles of the two soils obtained using the exact and boundary-layer solutions at different times. (a) Aeolian sandy soil, 1 h; (b) aeolian sandy soil, 5 h; (c) aeolian sandy soil, $10 \mathrm{~h}$; (d) paddy soil, $1 \mathrm{~h}$; (e) paddy soil, $3 \mathrm{~h}$; and (f) paddy soil, $5 \mathrm{~h}$. 
Table 1. Experimental setup and physical parameters of the soil columns.

\begin{tabular}{cccccc}
\hline Soil type & $\begin{array}{c}\text { Soil-column } \\
\text { height }(\mathrm{cm})\end{array}$ & $\begin{array}{c}\text { Average } \\
\text { pore-water } \\
\text { velocity }(\mathrm{cm} / \mathrm{h})\end{array}$ & $\begin{array}{c}\text { Bulk density } \\
\left(\mathrm{g} / \mathrm{cm}^{3}\right)\end{array}$ & $\begin{array}{c}\text { Water head } \\
(\mathrm{cm})\end{array}$ & Probe number \\
\hline Aeolian sandy soil & 68 & 1.05 & 1.34 & 5.50 & 6 \\
Paddy soil & 36 & 2.46 & 1.22 & 8.30 & 4 \\
\hline
\end{tabular}

Table 2. Relative errors of the logarithmic model with different transport parameters at a time of $3 \mathrm{~h}$.

\begin{tabular}{|c|c|c|c|c|c|}
\hline & $v$ & $\lambda=1$ & $\lambda=10$ & $\lambda=30$ & $\lambda=50$ \\
\hline \multirow{5}{*}{$R=1$} & 0.02 & 0.0778 & 0.0906 & 0.0939 & 0.0950 \\
\hline & 0.2 & 0.1026 & 0.0778 & 0.0847 & 0.0875 \\
\hline & & & & & \\
\hline & 2 & 0.2989 & 0.1026 & 0.0812 & 0.0749 \\
\hline & 20 & 0.5311 & 0.2989 & 0.1845 & 0.1430 \\
\hline \multirow{4}{*}{$R=20$} & 0.02 & 0.0929 & 0.0969 & 0.0977 & 0.0980 \\
\hline & 0.2 & 0.0823 & 0.0929 & 0.0953 & 0.0961 \\
\hline & 2 & 0.0812 & 0.0823 & 0.0884 & 0.0910 \\
\hline & 20 & 0.2236 & 0.0812 & 0.0753 & 0.0778 \\
\hline \multirow{4}{*}{$R=50$} & 0.02 & 0.0950 & 0.0976 & 0.0981 & 0.0983 \\
\hline & 0.2 & 0.0875 & 0.0950 & 0.0966 & 0.0971 \\
\hline & 2 & 0.0749 & 0.0875 & 0.0920 & 0.0935 \\
\hline & 20 & 0.1430 & 0.0749 & 0.0804 & 0.0837 \\
\hline
\end{tabular}

Table 3. $D$ and $R$ for the soil-column experiments estimated using different models.

\begin{tabular}{ccccc}
\hline & \multicolumn{2}{c}{ Aeolian sandy soil } & \multicolumn{2}{c}{ Paddy soil } \\
\cline { 2 - 5 } & $D\left(\mathrm{~cm}^{2} / \mathrm{h}\right)$ & $R$ & $D\left(\mathrm{~cm}^{2} / \mathrm{h}\right)$ & $R$ \\
\cline { 2 - 5 } Logarithmic model & 1.050 & 0.874 & 3.809 & 1.028 \\
Polynomial model & 2.260 & 2.440 & 8.916 & 2.808 \\
CXTFIT & 2.093 & 0.931 & 2.262 & 0.900 \\
\hline
\end{tabular}




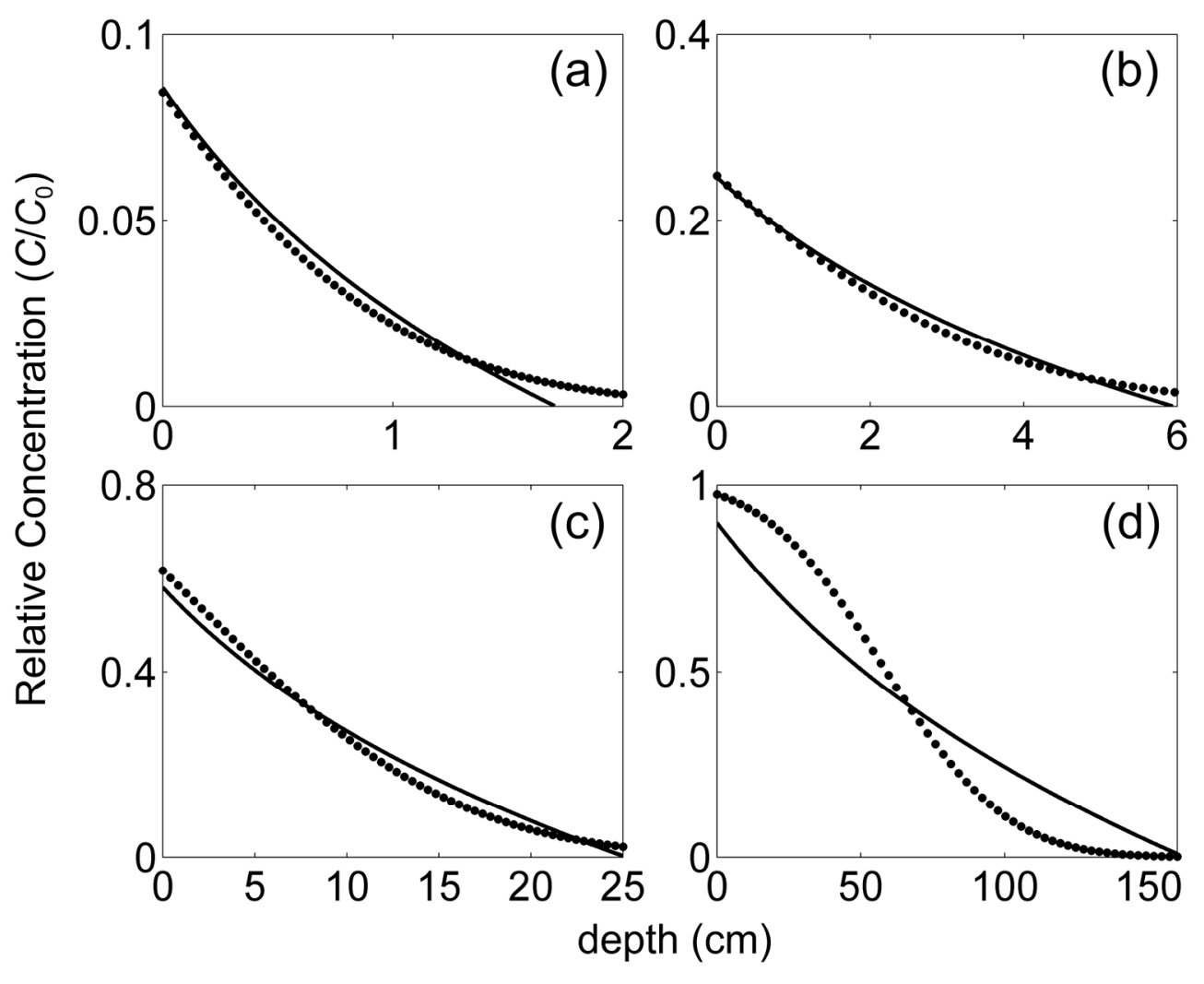

—Logarithmic profile ..... Exact profile

$181 \times 158 \mathrm{~mm}(300 \times 300 \mathrm{DPI})$ 


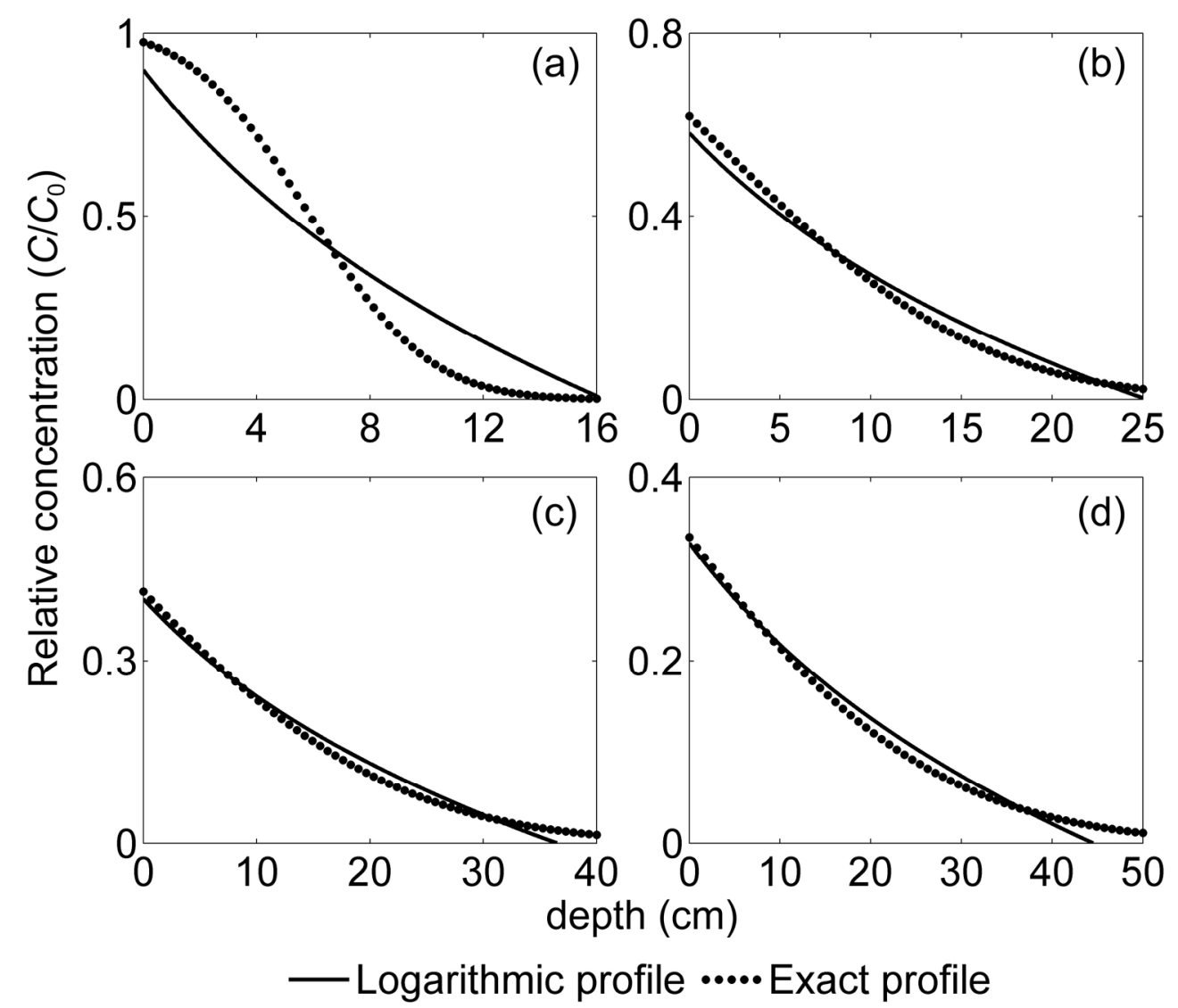

$176 \times 153 \mathrm{~mm}(300 \times 300 \mathrm{DPI})$ 


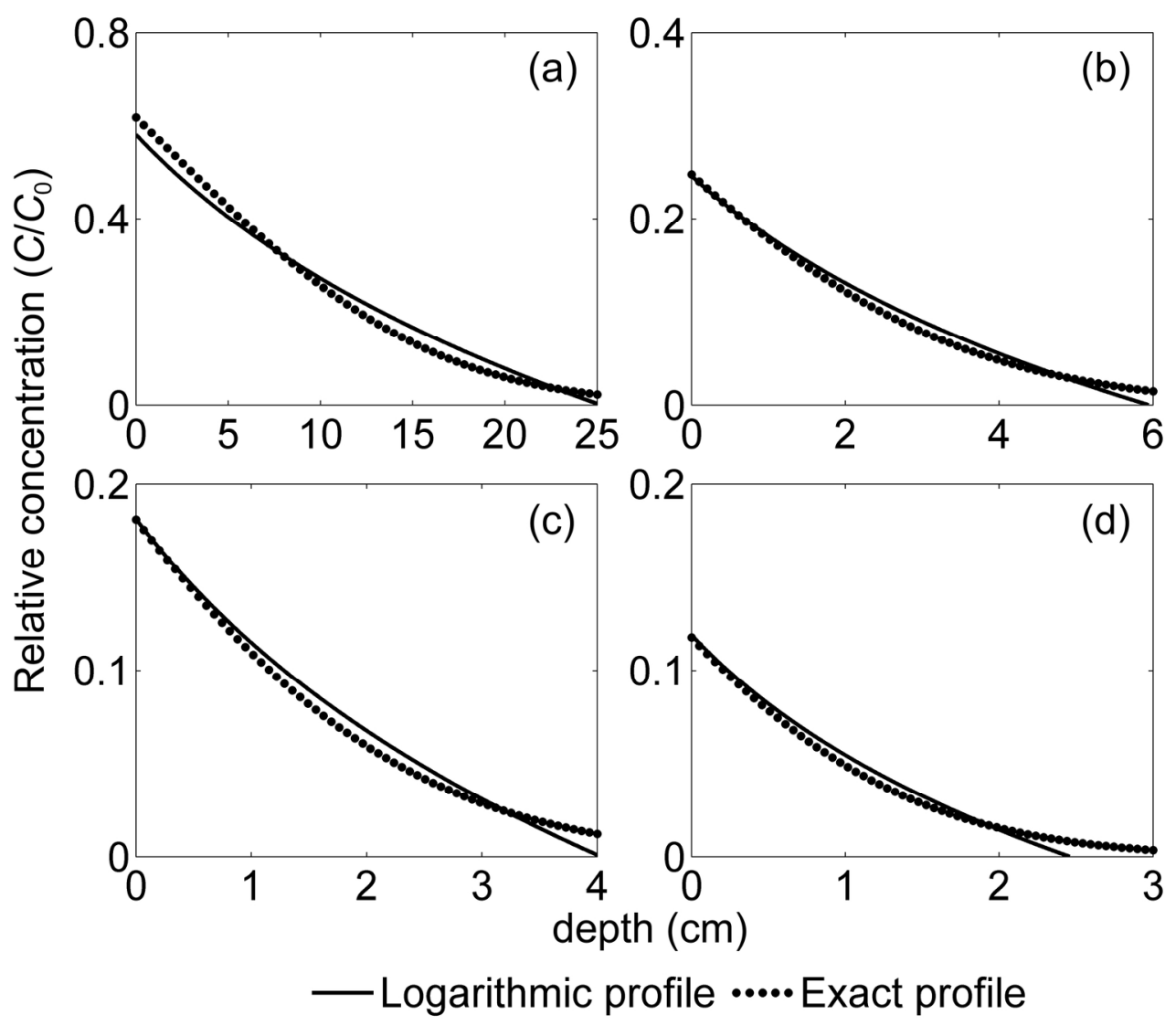

$176 \times 152 \mathrm{~mm}(300 \times 300 \mathrm{DPI})$ 


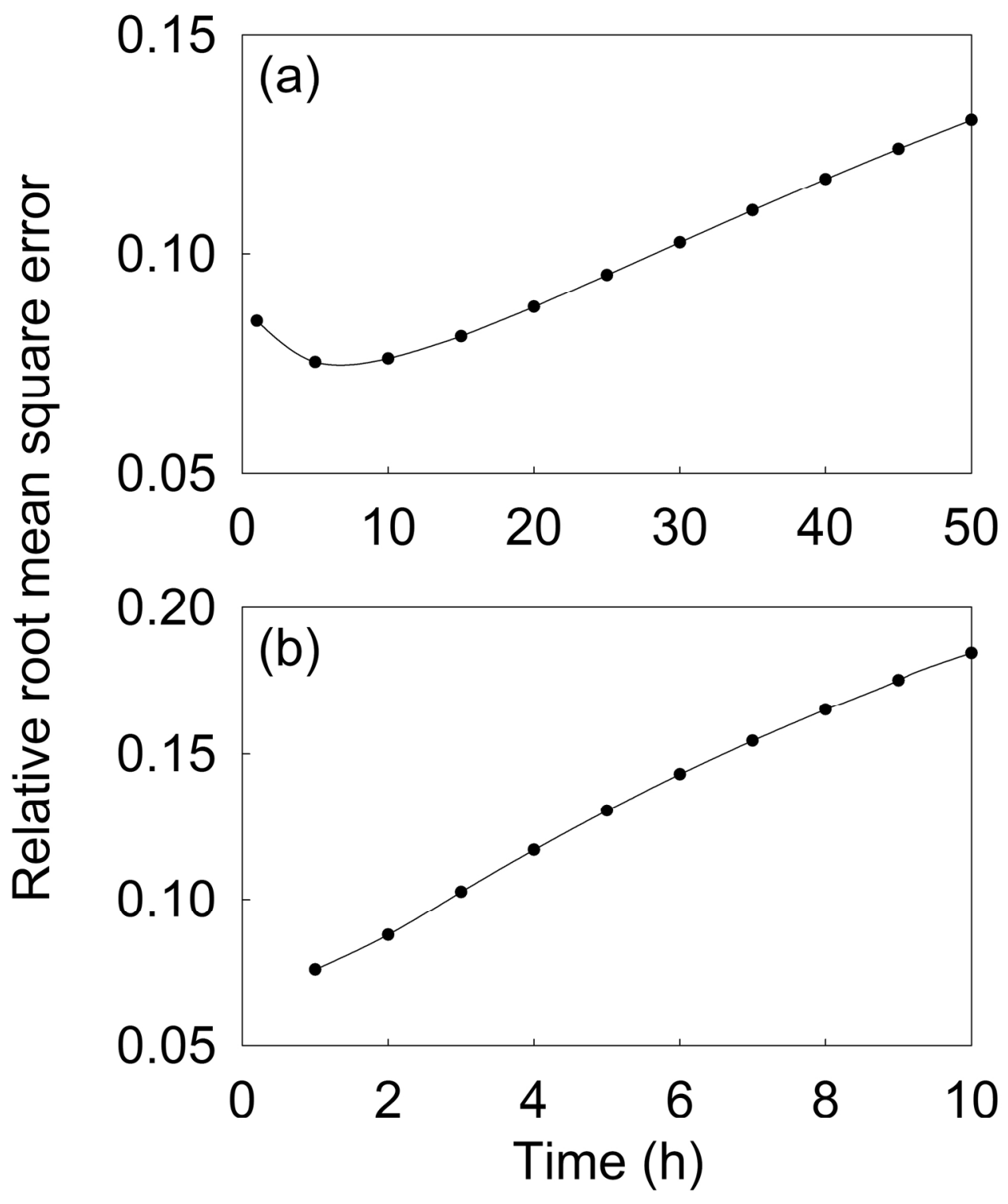

$169 \times 202 \mathrm{~mm}(300 \times 300$ DPI $)$ 


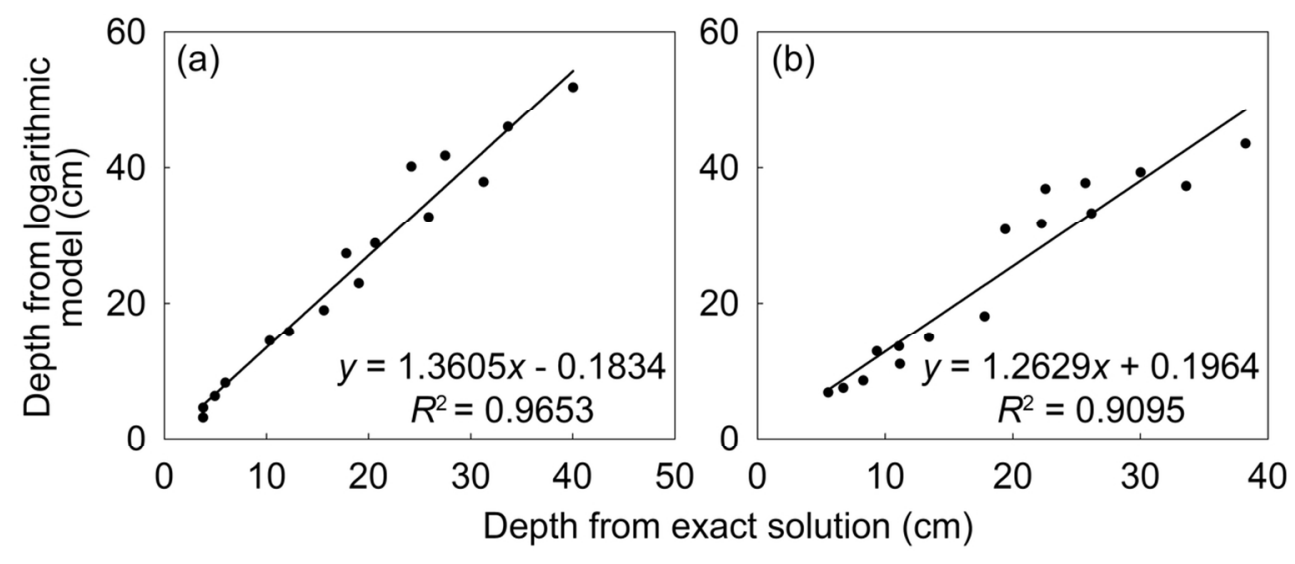

$109 \times 47 m m(300 \times 300$ DPI $)$ 


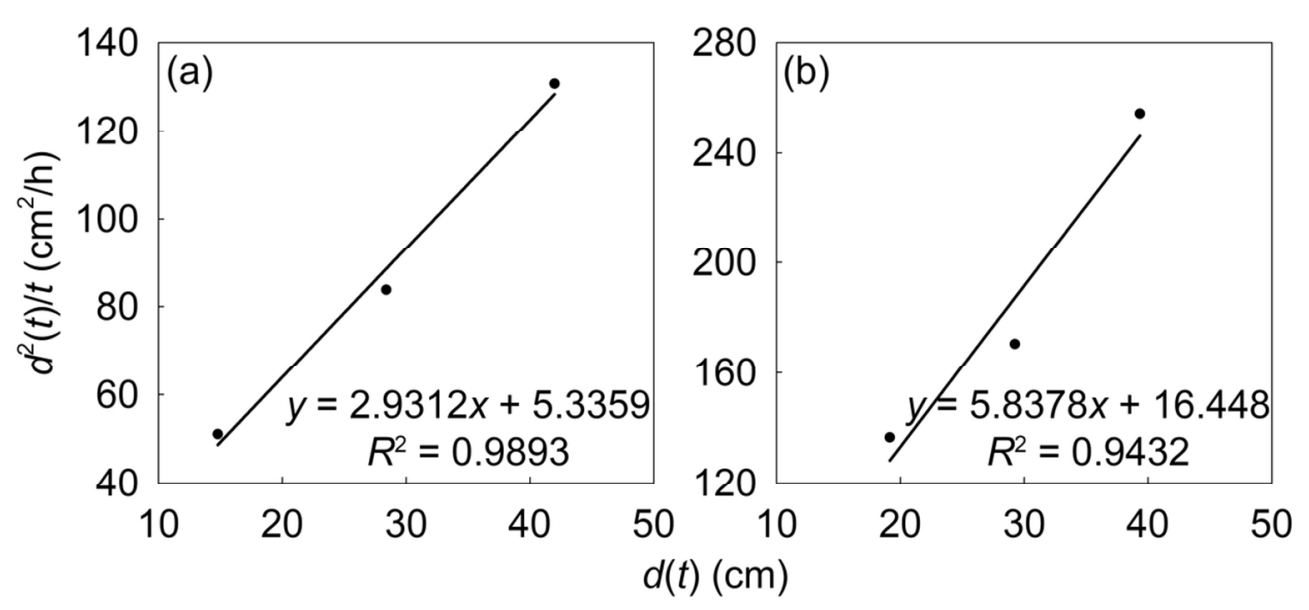

$106 \times 48 \mathrm{~mm}(300 \times 300$ DPI $)$ 


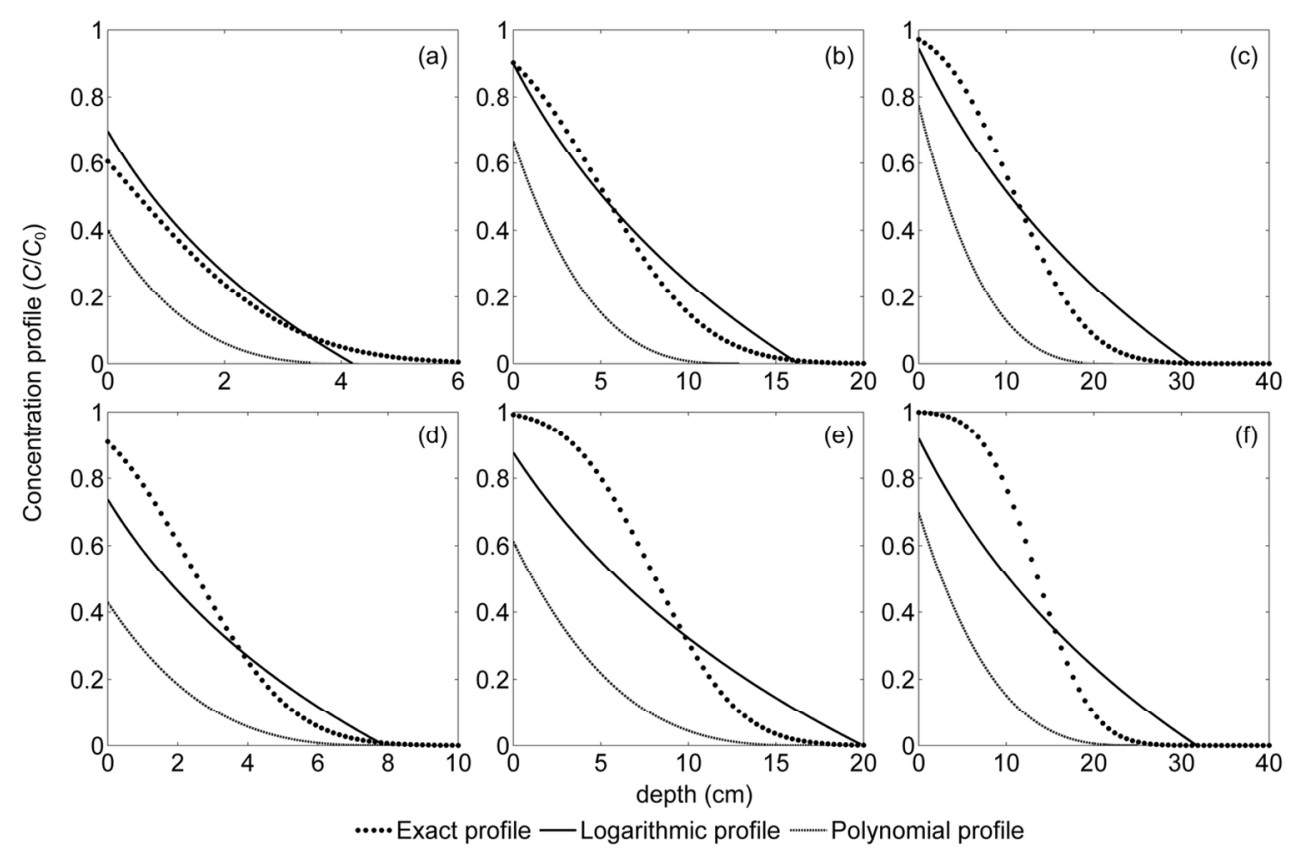

$128 \times 82 \mathrm{~mm}(300 \times 300$ DPI $)$ 